\title{
Agricultura familiar, segurança e soberania alimentar e nanotecnologia: onde estamos, para onde vamos
}

Paulo Roberto Martins ${ }^{1}$

\section{Resumo}

Em continuidade a reflexão realizada em 2007/2008 e publicada em 2009 por Martins et al., "Impactos das Nanotecnologias na cadeia de produção da soja brasileira”, o presente artigo tem por objetivo introduzir a questão do desenvolvimento das nanotecnologias e suas relações com agricultura familiar e a segurança e soberania alimentar no Brasil. Um panorama geral será apresentado em termos de como estamos no desenvolvimento das nanotecnologias aplicadas à agricultura. Apresenta-se quem no Brasil está trabalhando no desenvolvimento dessa tecnologia aplicada à agricultura, bem como será apresentada de forma sintética o que ocorre no panorama global. A partir dos elementos anteriormente indicados e de princípios adotados neste trabalho relativos ao tema segurança e soberania alimentar será apresentado uma análise em termos prospectivos dos possíveis impactos do desenvolvimento das nanotecnologias aplicadas à agricultura em suas relações com a agricultura familiar brasileira e também os possíveis impactos nas questões relativas à segurança e soberania alimentar posta pelo desenvolvimento dessa tecnologia no caso brasileiro. Ao final apresentam-se algumas conclusões preliminares para a discussão pública.

Palavras-chave: nanotecnologias, desenvolvimento, agricultura familiar, segurança alimentar, soberania alimentar. 


\title{
Familiar agriculture, food security and sovereignty and nanotechnology: where we are, where are we going
}

\begin{abstract}
Continuing the reflection carried out in 2007/2008 and published in 2009 by Martins et all, "Impacts of Nanotechnology in the production chain of Brazilian soy" , the present article aims to introduce the issue the development of nanotechnologies and their relationships with family agriculture and food security and sovereignty in Brazil. An overview will be presented in terms of how we are in the development of nanotechnologies applied agriculture. It is presented in Brazil who is working on the development of this technology applied to agriculture, and will be presented in summary form what happens in the global landscape. From the above listed elements and principles adopted in this paper on the subject security and sovereignty feeds will be presented an analysis in prospective terms of the possible impacts of the development of nanotechnologies applied to agriculture in its relations with the Brazilian family farming and also the possible impacts on issues related to food security and sovereignty posed by the development of this technology in the Brazilian case. At the end we present some preliminary findings for public discussion.
\end{abstract}

Keywords: nanotechnologies, development, familiar agriculture, food security, food sovereignty

\section{Introdução}

Em primeiro lugar cabe explicitar o que este artigo não pretende ser. Não pretende ser um artigo de referência no que tange a caracterização do que seja a agricultura familiar no Brasil, bem como do que seja segurança e soberania alimentar, isto porque 
há um contingente de trabalhos importantes produzidos sobre esses temas ao longo de décadas. Aqui iremos eleger algumas dessas contribuições, sem refazer o debate entre as diversas visões expressas pelos diversos trabalhos produzidos.

A decisão tomada e explicitada acima tem sua justificativa no que este artigo pretende ser. Pretende ser um artigo de referência sobre as relações entre o desenvolvimento de nanotecnologias no Brasil e a produção agrícola organizada por um conjunto de agricultores denominados de agricultores familiares, bem como as relações desta tecnologia ora em análise e a segurança e soberania alimentar no Brasil.

Nesse sentido, este artigo pretende ser inovador quanto ao tema proposto para análise e prospectivo no que tange ao futuro da agricultura familiar e da segurança e soberania alimentar, tendo em vista o desenvolvimento das nanotecnologias no Brasil.

Com isso, este artigo pretende despertar a atenção de outros pesquisadores para com esse tema de pesquisa e também despertar nos formuladores de políticas públicas relativas à agricultura familiar e à segurança e soberania alimentar a importância de levar em conta a introdução destas tecnologias / nanotecnologias no Brasil, com seus impactos nos temas objetos desta análise.

\section{N anotecnologia: conceitos e def inições}

O que o leitor verá a seguir está baseado no livro "Impactos das Nanotecnologias na Cadeia de Produção da Soja Brasileira”. Esse livro é o produto de uma pesquisa realizada com o apoio do NEAD/MDA $\left({ }^{2}\right)$.

2 MARTINS, Paulo R e Ramos, SORAIA F. (org). Impactos das nanotecnologias na cadeia de produção da soja brasileira. São Paulo: Xamã Editora, 2009, pp. 25-31 
Existe uma vasta literatura que apresenta conceitos e definições do que sejam as nanotecnologias. Segundo o Center for Responsible Nanotechnology, por exemplo, uma definição básica é que a nanotecnologia é a engenharia de sistemas funcionais na escala molecular. Em seu sentido original, nanotecnologia refere-se a projetada habilidade de construir coisas de "baixo para cima" (botton up), utilizando técnicas e ferramentas que estão sendo desenvolvidas atualmente para fazer produtos acabados e de alta performance (Center for Responsible / Technology.)

De acordo com o grupo canadense Erosion, Technology Concentration (ETC): As nanotecnologias referem-se à manipulação da matéria em escala de nanômetros (um bilionésimo de metro). A ciência em nanoescala opera no campo de um único átomo e moléculas. $\mathrm{Na}$ atualidade, as nanotecnologias comerciais envolvem materiais científicos, ou seja, materiais que são produzidos por pesquisadores aptos e que são mais resistentes e duráveis, utilizando a vantagem que resulta da alteração que ocorre nas suas propriedades quando as substâncias são reduzidas à dimensão de nanoescala. No futuro, quando a auto replicação molecular, em nível de nanoescala, se tornar uma realidade comercial, as nanotecnologias caminharão para a manufatura convencional. Enquanto as nanotecnologias oferecem oportunidades para a sociedade, elas também podem trazer profundos riscos sociais e ambientais não apenas por ser uma tecnologia capacitadora de tecnologias para a indústria biotécnica, mas também porque ela envolve a manipulação atômica que poderá tornar possível a fusão do mundo biológico com o mecânico. Há uma necessidade urgente para se avaliar as implicações sociais de todas as nanotecnologias [...]. (ETCGROUP, 2004)

A nanotecnologia pode ser apresentada em duas formas. Na primeira delas, a tecnologia caracteriza-se por dois aspectos principais: 1) o prefixo "nano", que é indicador de medida: 1 nano significa a bilionésima parte de um metro, ou seja, 10-9 metros. Nesse caso, nanotecnologia refere-se somente à escala, e não a objetos; 
2) refere-se a uma série de técnicas utilizadas para manipular a matéria na escala de átomos e moléculas que, para serem enxergadas, requerem microscópios especiais, muito potentes.

A título de ilustração, pode-se citar que um único fio de cabelo humano tem a dimensão de cerca de 80 mil nanômetros (nm) de espessura, enquanto $1 \mathrm{~nm}$ contém 10 átomos de hidrogênio colocados lado a lado. A conhecidíssima molécula de DNA tem o tamanho de aproximadamente 2,5 nm de largura, enquanto um glóbulo vermelho tem $5 \mathrm{mil} \mathrm{nm}$ de diâmetro. Ou, ainda ilustrativamente, um nanômetro corresponderia ao tamanho de uma bola de futebol em relação ao globo terrestre.

A segunda forma de se apresentar a nanotecnologia consiste em considerar a nanociência como o estudo dos princípios fundamentais de moléculas e estruturas com uma dimensão entre 1 a 100 nm (nanômetros). A nanotecnologia seria, então, a aplicação dessas moléculas em nanoestruturas, ou dispositivos nanométricos.

As partículas nano, embora sendo do mesmo elemento químico, comportam-se de forma distinta em relação às partículas maiores, em termos de cores, propriedades termodinâmicas, condutividade elétrica, etc. Portanto, o tamanho da partícula é de suma importância em relação aos efeitos que podem produzir, e porque muda a natureza das interações das forças entre as moléculas do material e, assim, muda os impactos que estes processos ou produtos nanotecnológicos podem causar ao meio ambiente, à saúde humana e à sociedade como um todo.

Mas, como se criam as nanoestruturas com objetivos industriais? Duas são as técnicas para se criarem nanoestruturas, com variados níveis de qualidade, velocidade e custos. Elas são conhecidas como bottom up (de baixo para cima) e top down (de cima para baixo). É preciso realçar que no início do século XXI a tendência de convergência entre essas técnicas está em curso. 
A técnica bottom up proporciona a construção de estruturas átomo por átomo ou molécula por molécula, mediante três alternativas:

1) síntese química (chemical synthesis), em geral utilizada para produzir matérias-primas, nas quais são utilizadas moléculas ou partículas nano;

2) auto-organização (self assembly). Nessa técnica, os átomos ou moléculas organizam-se de forma autônoma por meio de interações físicas ou químicas, construindo, assim, nanoestruturas ordenadas. Diversos sais em formas de cristais são obtidos por esta técnica;

3) organização determinada (positional assembly). Nesse caso, átomos e moléculas são deliberadamente manipulados e colocados em determinada ordem, um por um.

Já a técnica top down tem por objetivo reproduzir algo, porém em menor escala que o original e com maior capacidade de processamento de informações, como em um chip, por exemplo. Isso é feito mediante dois caminhos: engenharia de precisão ou litografia. A indústria de semicondutores vem realizando isso nos últimos 30 anos.

A ideia de que a matéria é composta por átomos já tem cerca de 2.400 anos, época em que o filósofo grego Demócrito defendia esta tese. Mas somente no final da década de 1950 é que ocorreu um fato que marcou o início da nanotecnologia em nossos tem$\operatorname{pos}^{3}$ : Richard Feynman (1960) afirmou que "Os princípios da física não falam contra a possibilidade de se manipular as coisas

30 físico estadunidense Richard Phillips Feynman (11/5/1918 - 15/2/1988) fez uma conferência no dia 29 de dezembro de 1959, em uma reunião da Sociedade Americana de Física realizada no Instituto de Tecnologia da Califórnia (Caltech), denominada There's plenty of room at the bottom (há muito espaço lá em baixo). A primeira publicação dessa conferência deu-se em fevereiro de 1960 no Caltech's Engineering and Science. 
átomo por átomo". Apontou, também, para o que seria, a seu ver, a principal barreira para a manipulação na escala nanométrica: a impossibilidade de vê-la.

Em 10 de agosto de 1982, 23 anos após a palestra de Feynman, a IBM conseguiu a patente do denominado microscópio de varredura de tunelamento eletrônico (scanning tunneling microscope - STM), que permite a visualização de imagens em tamanho nano. A partir desse microscópio outro foi desenvolvido, levando o nome de microscópio de microssondas eletrônicas de varredura (scanning probe microscope - SPM), que permite visualizar e manipular átomos e moléculas.

O termo nanotecnologia foi utilizado primeiramente pelo professor Norio Taneguchi, da Universidade de Ciência de Tóquio. Ele usou esse termo para descrever a fabricação precisa de novos materiais com tolerâncias nanométricas.

Nos anos 1980, nano adquiriu nova conotação devido à publicação do livro de K. Eric Drexler (1986) intitulado Engines of creation: the coming era of nanotechnology. Em 1992, com a publicação da tese de doutorado do mesmo autor $\left({ }^{4}\right)$, intitulada Nanosystems: molecular machinery, manufacturing and computation, a nanotecnologia ganhou novo impulso na comunidade científica.

\subsection{0 debate sobre os possíveis horizontes das nanotecnologias}

Este debate tem como referência a questão relativa a dois tipos de inovação presentes no desenvolvimento científico e tecnológico, a saber: inovações incrementais e revolucionárias.

As inovações incrementais ocorrem constantemente, segundo o ritmo de cada setor, consistindo em simples melhoria da gama

4 Tese defendida no Massachusetts Institute of Technology (MIT). 
de produtos e de processos existentes (inovação marginal ou secundária). No limite das aplicações das nanotecnologias, entretanto, poderão assumir características revolucionárias.

As inovações revolucionárias não se limitam a criar novos produtos e processos, mas originam uma série de novas atividades, afetando todos os segmentos econômicos e alterando a estrutura de custos dos meios de produção e de distribuição.

A bibliografia em nanotecnologia já é bastante intensa e heterogênea. Para uma síntese do debate, pode-se utilizar o trabalho de Wood, Jones e Geldart (2003). Em grandes blocos, o debate pode ser referenciado em termos dos que acreditam ser a nanotecnologia portadora de radical descontinuidade, enquanto os opositores a esta ideia advogam que a nanotecnologia apresenta somente uma continuidade evolucionária de outras tecnologias. Entre esses dois extremos também existem vários autores.

Entre os defensores da radical descontinuidade, podem-se citar K. Eric Drexler, Jamie Dinkelacker, The Foresight Institute, Bill Joy, Glenn Harlan Reynolds, Damien Broderick, Mark Suchman, Mihail Rocco. Esse conjunto de autores pode ser denominado de "nano-otimista".

No campo oposto, se tem os evolucionaristas, cujos expoentes, entre outros, são George M. Whiteside, Richard E. Smalley, Philip Ball, Denis Laveridge, Gary Stix. Esses podem ser denominados de "nanopessimistas", na medida em que entendem que as nanotecnologias se encontram apenas no campo das inovações incrementais.

Numa versão sintética sobre esse tema nano-otimista/pessimista, Letham (apud Basl, 2005) analisou as implicações sociais das nanotecnologias. Ele descreveu dois paradigmas de pensamento: um que chamou de paradigma otimista, definido pela crença de que benefícios das nanotecnologias superam seus riscos, e 
outro que chamou de paradigma pessimista, definido pela crença de que os riscos das nanotecnologias são maiores do que os benefícios. Entre os dois grupos acima comentados estão as instituições promotoras da nanotecnologia e os comentadores de tecnologia.

Segundo Wood, Jones e Geldart (2003), as entidades promotoras localizam-se em diversos países e em indústrias, como o Departamento de Comércio e Indústria da Inglaterra, a Direção de Tecnologias Industriais da Comissão Europeia, a National Nanotechnology Initiative e a National Science Foundation, ambas do governo estadunidense.

Entre os comentadores, os autores indicam o mais importante deles: a ONG canadense ETCGroup, além de Debra R. Rolinson, do Laboratório de Pesquisa Naval, e Vick Colvin, da Rice University, ambos nos Estados Unidos

Desse rol de autores e instituições indicados, detalham-se um pouco mais as contribuições do professor Mark Suchman e do ETCGroup. Essas ideias encontram-se expostas de maneira ampla em Martins (2005) e ETCGroup (2005).

As controvérsias relativas à nanotecnologia podem ser captadas nos diversos trabalhos do ETCGroup, em especial em "Nanotecnologia: os riscos da tecnologia do futuro" (2005), no qual uma síntese dos diversos problemas é apresentada, a começar pelo impacto dessa tecnologia nas economias dos países do Hemisfério Sul, na vida das pessoas, na segurança, na saúde humana, no meio ambiente, nos direitos humanos, nas políticas sociais, na agricultura, nos alimentos. Esse trabalho apresenta quem tem o controle dessa tecnologia (as grandes empresas) e a quanto chegam os investimentos nesta tecnologia (US\$ 8,6 bilhões).

Em suas recomendações, o ETCGroup (2005) afirma que: ao permitir que produtos da nanotecnologia cheguem ao mercado 
na ausência de debate público e sem regulação, os governos, o agronegócio e as instituições científicas já comprometeram o potencial das tecnologias em escala nanométrica de serem utilizadas de forma benéfica. 0 fato de não haver, atualmente, em qualquer parte do mundo, normas de regulação para avaliar novos produtos em escala nanométrica na cadeia alimentar, representa uma inaceitável e culposa negligência. [...] Devem ser tomadas medidas para restaurar a confiança nos sistemas alimentares e para se ter certeza de que as tecnologias em escala nanométrica, se introduzidas, sejam feitas sob rigorosos padrões de saúde e segurança.

Em relação às aplicações das nanotecnologias, podem-se considerar dois enfoques: 1) de uma nanoestrutura passiva com novas propriedades e funções para uma mesma composição química; nanoestruturas inertes ou reativas que apresentam comportamento estável e propriedades quase constantes durante seu uso; 2) de transição para nanoestruturas ativas, em que sucessivas mudanças podem ocorrer de modo planejado ou imprevisto no ambiente.

Essas observações deverão ser consideradas quando do uso das nanotecnologias na agricultura em geral e, em especial, na produção de alimentos.

\section{Segurança e soberania alimentar: breves conceitos}

A opção foi adotar neste trabalho a forma oficial/governamental de entender o que seja a segurança e a soberania alimentar. Isso se justifica porque as análises relativas ao desenvolvimento das nanotecnologias serão realizadas em função das ações realizadas pelo governo federal ao longo deste Século XXI. Assim sendo, estaremos usando tanto para o campo da segurança e soberania alimentar e nanotecnologia as formas oficiais de entendimento destes temas. Assim sendo, entendemos: 
A Segurança Alimentar, enquanto estratégia ou conjunto de ações, deve ser intersetorial e participativa, e consiste na realização do direito de todos ao acesso regular e permanente a alimentos de qualidade, em quantidade suficiente, sem comprometer o acesso a outras necessidades essenciais, tendo como base práticas alimentares promotoras da saúde, que respeitem a diversidade cultural e que sejam ambiental, cultural, econômica e socialmente sustentáveis.

O modelo de produção e consumo de alimentos é fundamental para garantia de segurança alimentar, pois, para além da fome, há insegurança alimentar e nutricional sempre que se produz alimentos sem respeito ao meio ambiente, com uso de agrotóxicos que afetam a saúde de trabalhadores/as e consumidores/as, sem respeito ao princípio da precaução, ou, ainda, quando há ações, incluindo publicidade, que conduzem ao consumo de alimentos que fazem mal à saúde ou ao distanciamento de hábitos tradicionais de alimentação.

A segurança alimentar e nutricional demanda ações intersetoriais de garantia de acesso à terra urbana e rural e território, de garantia de acesso aos bens da natureza, incluindo as sementes, de garantia de acesso à água para consumo e produção de alimentos, da garantia de serviços públicos adequados de saúde, educação, transporte, entre outros, de ações de prevenção e controle da obesidade, do fortalecimento da agricultura familiar e da produção orgânica e agroecológica, da proteção dos sistemas agroextrativistas, de ações específicas para povos indígenas, populações negras e povos e comunidades tradicionais. É, ainda, fundamental que as ações públicas para garantia de segurança alimentar possam contemplar abordagem de gênero e geracional.

A soberania alimentar é um princípio crucial para a garantia de segurança alimentar e nutricional e diz respeito ao direito que tem os povos de definirem as políticas, com autonomia sobre o que produzir, para quem produzir e em que condições produzir. Soberania alimentar significa garantir a soberania dos agriculto- 
res e agricultoras, extrativistas, pescadores, entre outros grupos, sobre sua cultura e sobre os bens da natureza ${ }^{5}$.

\section{0 contexto das relações entre segurança e soberania alimentar e nanotecnologia}

Neste tópico o diálogo será realizado com o texto "A Agricultura e a Promoção da Soberania e Segurança Alimentar e Nutricional: Entraves e Desafios" de autoria de Renato S. Maluf (2013). As reflexões realizadas servirão de guia e serão relacionadas ao tema do desenvolvimento das nanotecnologias e seus impactos nas questões relativas à segurança e soberania alimentar.

A primeira questão indicada por Maluf (2013) é que o Brasil está integrado ao sistema alimentar globalizado, sendo um player importante neste mercado, como grande exportador de commodities agrícolas e produtos agroalimentares semiprocessados. Assim sendo, é preciso entender a participação da agricultura brasileira na denominada "segurança alimentar global" em tempos de crise do próprio sistema alimentar.

Dentro desse contexto, o autor em questão elege duas questões centrais: a) coexistência de diferentes modelos de agricultura no meio rural brasileiro, uma das principais manifestações das tensões e contradições presentes em nossa sociedade; b) a inexistência de uma política nacional de abastecimento alimentar orientada pelo marco de referência capaz de articular a promoção da produção diversificada e sustentável de alimentos em bases familiares e com ampliação do acesso da população brasileira a uma alimentação adequada e saudável (Maluf, 2013).

5 http://www2.planalto.gov.br/consea/o-conselho/conceitos-1/direito-humano-aalimentacao-adequada. 
Em tópico mais a frente neste trabalho iremos debater o quanto o desenvolvimento das nanotecnologias no Brasil foi desenhado, colaborou para que as tensões e contradições no meio rural fosse sanado e/ou mitigado, assim como a contribuição para a produção diversificada e sustentável de alimentos em bases familiares. Terá o desenvolvimento das nanotecnologias no Brasil ter sido planejada/desenhada para contribuir com as questões colocadas por Maluf (2013) ou mesmo sem planejamento teria contribuído na direção de superar as questões levantadas pelo autor em questão?? É o que veremos mais adiante neste trabalho.

No artigo indicado, Maluf (2013) demonstra que a crise alimentar que abalou o mundo com intensas oscilações de preços internacionais de commoditties alimentares configura-se como uma crise sistêmica atrelada a três outras crises, a saber: crise econômica financeira, crise ambiental (climática) e crise energética. Para o autor, a agricultura está intrinsicamente entrelaçada com essas três crises. As causas que afetam o sistema alimentar global, algumas delas não tradicionais e com temporalidades distintas, atuam de forma combinada, conferindo caráter sistêmico à crise atual. Entre as causas, Maluf (2013) destaca: a) continua elevação da demanda de alimentos; b) quantidades crescentes de grãos básicos destinados à produção de biocombustíveis; c) elevação dos preços do petróleo; eventos climáticos extremos; d) especulação financeira com as commoditties alimentares atreladas aos demais mercados de ativos financeiros; e) um longo período de subinvestimento público na agricultura de base familiar ou camponês.

O autor em questão também ressalta que,

No entanto, não se tratando de um fenômeno meramente conjuntural, a crise alimentar tem acarretado mudanças importantes no sistema alimentar mundial em termos de localização da produção agrícola - não apenas pelas mudanças climáticas, mas como resultado de investimentos interna- 
cionais com acaparamento de terras (land grabbing). Fortaleceu-se o poder das grandes corporações internacionais nos principais componentes do sistema alimentar mundial, em particular, ampliando a parcela já majoritária das operações intrafirmas no comércio agroalimentar internacional. Não obstante sua importância, o comércio internacional tem um antigo histórico de fonte não confiável de segurança alimentar, característica reafirmada na recente crise (Maluf, 2013, p. 138-139).

É importante destacar que Maluf (2013) indica a necessidade da revisão de paradigmas tecnológicos responsáveis pela produção de alimentos, para não colocar em risco a sustentabilidade ambiental e a capacidade de renovação do planeta. Para esse autor, isso implica em valorizar o enfoque agroecológico. Isso também implica em uma valorização da agricultura familiar dada sua produção diversificada em oposição à monocultura em larga escala com uso intensivo de agrotóxicos e suas implicações, característica dos agronegócios. Aqui se coloca mais um reflexão em relação ao desenvolvimento das nanotecnologias no Brasil. 0 que esse desenvolvimento contribuiu no caminho relativo à agroecologia ou a opção deste desenvolvimento das nanotecnologias contribuiu foi para produzir conhecimentos, processos e produtos que atenderam mais o agronegócio que a agroecologia?

\section{Reflexões sobre abastecimento, modelos de agricultura e nanotecnologia}

Em continuidade na interação com o texto de Maluf (2013) já citado, cabe apresentar dentro das discussões propostas por este autor no que se refere a abastecimentos e agricultura, os principais conceitos, ideias, propostas e as questões as quais iremos refletir em relação ao desenvolvimento das nanotecnologias no Brasil.

Maluf (2013) começa essa parte de seu texto fazendo uma afirmação e apresentando um conceito: 
Pode-se afirmar que o Brasil abdicou de adotar uma política soberana de abastecimento, opção consagrada e aprofundada na década de 1990. As inflexões havidas nas políticas agroalimentares e o acionamento de alguns instrumentos a partir do governo Lula (2003) não permitem afirmar que aquela opção tenha sido revertida e uma política de abastecimento propriamente dita esteja em curso. No entanto, na construção social antes apresentada, o abastecimento alimentar, quando devidamente enfocado, aparece como questão estratégica por ser o elo de ampliação do acesso a uma alimentação adequada e saudável e à promoção da produção de alimentos de base familiar, diversificada e sustentável, notadamente em sua etapa agrícola. (p. 146)

O que realçamos acima indica a importância da produção de alimentos de base familiar e sustentável, que na sua etapa agrícola está diretamente relacionada às tecnologias disponíveis a estes produtores. Como ressalta Maluf (2013), "Os modelos de produção e os tipos de alimentos interessam tanto ou mais que a mera disponibilidade de bens" (IBDEM, IDEM, p. 146). Qual tem sido a contribuição do desenvolvimento das nanotecnologias no Brasil em relação a este tipo de agricultura e a que tipos de produtos?

Maluf (2013) identifica na formação histórica brasileira a coexistência, no meio rural, da grande propriedade com diversas formas de pequenos e médios estabelecimentos agrícolas, resultando modelos distintos de agricultura e isto é uma das principais raízes de nossa desigualdade social. Está o desenvolvimento das nanotecnologias moldado, desenhado, produzido para combater esse tipo de desigualdade originado no meio rural brasileiro.

Para deixar claro a que tipos de agriculturas e atores sociais a pergunta acima é dirigida, apresentamos a seguir o conceito de agricultura familiar e agronegócio apresentada por Maluf (2013) no texto citado e que nos serve de guia nestas nossas reflexões: 
Desde meados da década de 1990, um conjunto bastante heterogêneo - por critérios como o nível de renda, bioma, sistemas de produção e fatores étnico-culturais - passou a se abrigar sob a noção de agricultura familiar, uma categoria sociopolítica que logrou reconhecimento legal e políticas públicas diferenciadas. Já o chamado agronegócio, termo usado para se referir à integração entre atividades agrícolas e não agrícolas (processamento dos produtos, comercialização etc.), se converteu em categoria político-ideológica representante da agricultura patronal. Não são dois mundos separados, é claro, mas que coexistem com complementaridades, conflitos e contradições. (Maluf, 2013, p.146-147).

O desenvolvimento das nanotecnologias privilegia um tipo de agricultura em detrimento de outro?? Quais atores oriundos desses tipos de agricultura estão a influenciar/determinar os rumos das nanotecnologias no Brasil no que tange a produção de conhecimentos voltados à agricultura? Em nota de rodapé - a seguir reproduzida - Maluf (2013) apresenta uma importante reflexão daquilo que ele denominou de "armadilha da modernização",

Em artigo anterior (Maluf, 2002), cunhei a expressão "armadilha da modernização" para fazer referência a um processo que, embora possa representar a emancipação de indivíduos, compromete a reprodução do grupo social como tal. Esta parece ser a marca da modernização agrícola, capaz de abastecer um país e gerar excedentes exportáveis com um número absoluto decrescente de agricultores em unidades produtivas especializadas de maior escala, quase sempre monocultoras, intensamente mecanizadas e dependentes de insumos químicos. (p.147).

Qual tem sido o papel do desenvolvimento das nanotecnologias no Brasil nesse processo de modernização / "armadilha de modernização"? O desenvolvimento das nanotecnologias tem ou não reforçado esse processo??

Em continuidade cabe apresentar as propostas elaboradas por Maluf (2013) em seu texto aqui analisado e indicar as reflexões que coloca em relação às nanotecnologias. 
Proposta 1. Organizar a oferta da produção oriunda da agricultura familiar, incentivar melhorias em qualidade, capilaridade e regularidade desta produção e promover a diversidade de produtos, formas de cultivo e hábitos alimentares. Nessa direção, contribuiria o aprimoramento da inserção no mercado pequeno varejo - setor pouco contemplado por políticas públicas no Brasil, em forte contraste com o antigo apoio conferido à expansão das grandes cadeias de varejo. Estimular as redes solidárias de produção, processamento, distribuição e consumo baseadas em empreendimentos associativos pode também ser um caminho promissor no campo agroalimentar.

Proposta 2. Programas de abastecimento podem contribuir para que os pequenos e médios produtores rurais e urbanos de alimentos, bem como o varejo de pequeno porte, aproveitem as oportunidades criadas pela segmentação dos mercados e diferenciação de produtos (produtos artesanais, orgânicos, com denominação de origem etc.). 0 desafio de construir mercados se coloca, principalmente, para a agricultura familiar, para a pequena indústria agroalimentar e para o varejo tradicional.

Em que as nanotecnologias desenvolvidas no Brasil estão a contribuir na execução dessas propostas ou pelo contrário, estão a indicar outros caminhos/propostas?

Em suas observações finais Maluf (2013) aponta,

os riscos da vinculação ao mercado global e a problemática dependência de uma oferta centralizada de alimentos, controlada por grande empresas corporativas. Segundo este autor, este diagnóstico impõe o resgate do papel do Estado e da participação social na busca de estratégias englobando: fortalecimento da agricultura camponesa e familiar; diversificação dos sistemas produtivos e de sua base genética; melhor aproveitamento de insumos e de fontes de energia localmente disponíveis; reestruturação dos sistemas nacionais de abastecimento com fortalecimento dos circuitos 
locais/regionais de produção, distribuição e consumo de alimentos; diversificação da cesta de consumo, valorizando a agricultura de base familiar igualmente diversificada, visando lograr, simultaneamente, uma dieta saudável e a atenuação dos impactos das elevações dos preços dos alimentos; ampliação da cooperação visando fortalecer estratégias regionais de abastecimento alimentar, especialmente nos países da América do Sul. (Maluf, 2013, p. 152).

E as nanotecnologias produzidas no Brasil têm apresentado alguma resposta, têm produzido algum conhecimento dirigido às questões acima elencadas? Enfim, qual tem sido a contribuição das nanotecnologias no Brasil, neste Séc. XXI, em relação ao tema da segurança e soberania alimentar? É o que veremos a seguir.

\section{Nanopartículas e macropoliticas: por onde vai 0 desenvolvimento das nanotecnologias no Brasil.}

Toda periodização utilizada para determinar quando algo surge na ciência e tecnologia de um país é problemática, por se tratar de um recorte da historia. Sempre é possível identificar que a origem de um evento remonta a um período anterior mais comumente admitido.

Também é preciso ressaltar que, quando se apresenta o desenvolvimento de um dado setor econômico ou de uma área de C\&T baseada apenas em fontes oficiais, certamente se apresenta parte do todo, ou seja, aquela parte que reflete apenas a visão oficial sobre o processo de desenvolvimento em questão.

Certamente, o Edital CNPq n.1 / 2001 que constituiu as primeiras quatro redes de pesquisas em nanotecnologia, tornou-se um marco do desenvolvimento desta tecnologia no Brasil, sendo importante ressaltar a articulação promovida em termos de recursos humanos e financeiros, neste caso no valor de $\mathrm{R} \$ 9.800 .000,00$ de reais, relati- 
vos ao período de 2001-2005. As quatro redes de pesquisas constituídas foram:

a) Nanobiotecnologia;

b) Nanodispositivos, Semicondutores e Materiais Nanoestruturados;

c) Materiais Nanoestruturados;

d) Nanotecnologia Molecular e de Interfaces.

Essa foi a primeira ação do Estado brasileiro centrado na organização das atividades de nanotecnologia. Seu objetivo era: fomentar a constituição e consolidação de Redes Cooperativas Integradas de Pesquisas Básica e Aplicada em Nanociência e Nanotecnologias, organizadas como centro virtuais de caráter multidisciplinar e abrangência nacional, doravante denominadas Redes, através de apoio a projetos de pesquisa cientifica e/ ou de desenvolvimento tecnológico em temas selecionados nas linhas de pesquisas em nanociências e nanotecnologias para 2001-2002 (6).

Em livro publicado em 2007, intitulado "Revolução Invisível. Desenvolvimento Recente da Nanotecnologia no Brasil", coordenado pelo autor deste trabalho, já indicava que: "É preciso explicitar que o caráter multidisciplinar atribuído à nanociência e à nanotecnologia nunca incorporou as ciências humanas, é que aquelas sempre foram entendidas e praticadas com a exclusão da área de humanidades" (Martins, 2007, pp. 12-13). Essa é a primeira exclusão aqui relatada e que permanece presente até o ano de escrita deste artigo, julho de 2013. 
0 ano de 2003, primeiro ano do governo do Presidente Luiz Inácio Lula da Silva, tinha como Ministro de Ciência e Tecnologia o cientista político Roberto Amaral, que instituiu a Coordenação Geral de Políticas e Programas em Nanotecnologia, marcando assim o inicio por parte do Estado da constituição de um aparato burocrático destinado à administrar e produzir políticas públicas em nanociência e nanotecnologia. Mediante a portaria 252 de 16/05/03 nomeou um grupo de trabalho composto exclusivamente por cientistas das áreas de exatas, com larga predominância dos físicos. Esse grupo foi constituído para "elaborar o Programa Nacional Quadrienal de Nanotecnologia" coordenado pelo físico Gilberto Fernandes de Sá. 0 documento produzido foi à consulta pública pela internet. Pela segunda vez, materializa-se a exclusão das ciências humanas desse processo, em que mais uma vez a concepção de multidisciplinariedade das nanotecnologias ignora aquela área das ciências, assim como também exclui a participação e controle social desse processo.

Outro fato importante nesse mesmo ano de 2003 foi a inclusão do "Programa de Desenvolvimento em Nanociência e Nanotecnologia" no Plano Pluri Anual, de 2004 a 2007, do Governo Federal. Programa que tem um aporte financeiro de $\mathrm{R} \$ 8.400 .000,00$ de reais para o período de 2004 a 2007. Assim sendo, o tema Nanociência e Nanotecnologia passa a fazer parte, pela primeira vez, deste instrumento obrigatório de planejamento (Plano Pluri Anual) que todo governo deve produzir no seu primeiro ano de mandato, por ser uma determinação constitucional.

Em 2004, O Presidente Lula resolve substituir o então Ministro de Ciência e Tecnologia Roberto Amaral pelo Deputado Eduardo Campos do Partido Socialista Brasileiro. Um grupo articulado de cientistas / físicos que estavam fora do governo e faziam criticas à condução do cientista politico Roberto Amaral no campo das nanotecnologias foram então incorporados aos quadros do MCT e passaram a dirigir os destinos da nanotecnologia no Brasil. 0 Físico Cylon Eudoxio Tricot Gonçalves da Silva passou a ser o 
Coordenador de Micro e Nanotecnologia do MCT, nova denominação da antiga Coordenação Geral de |Politicas e Programas em Nanotecnologia.

A primeira ação desse coordenador foi ignorar todas as contribuições públicas encaminhadas via consulta pública sobre o texto "Programa Nacional Quadrienal de Nanotecnologia"7. Colocou esse programa na gaveta e passou a produzir outro programa. Assim sendo, articulou a constituição da Rede Brasil Nano como um dos elementos do Programa de Desenvolvimento das Nanociências e Nanotecnologia, no âmbito da Politica Industrial e Tecnológica e de Comércio Exterior, que passou a ser regido pelas normas da Portaria MCT N. 614 de 1/12/2004.

Todo o processo de produção dessa portaria acima indicada foi caracterizado pela consulta reservada a alguns pesquisadores (portanto, consulta privada, não pública), entre os quais o autor deste paper, que não teve nenhuma de suas contribuições acatada pelo novo coordenador de micro e nanotecnologia $\left({ }^{8}\right)$. Isso se deu também com outros pesquisadores que dedicaram seu tempo de trabalho a produzir sugestões que foram ignoradas.

Em 7 de Abril de 2005, o Ministro de Ciência e Tecnologia Eduardo Campos assina a portaria de numero 192, designando os membros do conselho diretor da Rede Brasil Nano.

Aqui se materializa mais uma vez a exclusão. Nesse conselho, os representantes dos pesquisadores nacionais e internacionais nomeados não contemplam as ciências humanas, o setor indus-

7 As contribuições do autor se encontram registradas no Livro "Revolução Invisível. Desenvolvimento recente da Nanotecnologia no Brasil. São Paulo, Xama Editora, 2007, pp. 55-89

8 As contribuições do autor encontram registradas no Livro "Revolução Invisível. Desenvolvimento recente da Nanotecnologia no Brasil”. São Paulo, Xama Editora, 2007, pp. 23-33 
trial é contemplado com representação, mas os trabalhadores e consumidores não tiveram nenhum representante nomeado. Portanto, esse foi um conselho que contou apenas com representes do governo federal, da academia (excluindo a ciências humanas) e do setor privado patronal.

Ainda em 2004, o CNPq publicou o edital de n.13 aberto para pesquisas sobre os impactos sociais, ambientais e éticos da nanotecnologia. Esse foi o único edital aberto sobre a temática até julho de 2013. O Valor previsto para esse edital foi de $\mathrm{R} \$ 200.000,00$, dos quais foram usados apenas $\mathrm{R} \$ 125.000,00$, não sendo possível identificar até o presente em que foi aplicado os restantes $\mathrm{R} \$ 75.000,00$ não usados neste edital.

Para efeito de comparação, o edital CNPq 12/2004, publicado simultaneamente ao edital de n.13/2004 direcionados a projetos de nanobiotecnologia, tinha recursos previstos de $\mathrm{R} \$ 3.500 .000,00$. Outra comparação possível relativa ao edital $13 / 2004$ trata-se de relacionar os valores aplicados na produção de conhecimentos relativos à ciência da produção e a ciências dos impactos.

Para o leitor que quiser fazer as contas de maneira exata basta dividir o valor aplicado no único edital $(13 / 2004)$ para estudos de impactos da nanotecnologia no valor de $\mathrm{R} \$ 125.000,00$ e dividir pela soma dos valores relativo aos orçamentos de 2004 a 2011 que chegaram cerca de $\mathrm{R} \$ 75$ milhões. O que fica patente sem qualquer dúvida é que foi menos que 1\% (tendendo a zero) o dinheiro público aplicado na produção deste tipo de conhecimento (ciência dos impactos). 0 "outro lada da moeda" é que 99\% dos recursos públicos foram aplicados na geração de conhecimento relativo à ciência da produção. Portanto, fica assim demonstrado a imensa exclusão praticada por esta política pública de desenvolvimento das nanotecnologias no Brasil, no período de 2001 a 2011, no que toca a que tipos de conhecimentos (produção x impactos) foram produzidos. 
A intensificação dessa situação foi produzida ao longo dos anos de 2005/2009, pois o Edital CNPq 29/2005, que previa a construção de 10 redes cooperativas de pesquisas (em substituição as 4 redes decorrentes do Edital CNPq 01/2001), que estão abaixo indicadas:

1) Rede de nanofotônica

2) Rede de pesquisas em nanobiotecnologia e sistemas nanoestruturados

3) Rede de nanotecnologia molecular e de interfaces

4) Rede de nanotubos de carbono: ciência e aplicação

5) Rede de nanocosmêticos: do conceito às aplicações tecnológicas

6) Rede de microscopia de varredura eletrônica

7) Rede de pesquisas em simulação e modelagem de nanoestruturas

8) Rede cooperativa de pesquisa em revestimentos nanoestruturdos

9) Rede de pesquisa em nanoglicobiotecnologia

10) Rede de nanobiomagnetismo

Conforme se pode notar nos nomes das redes acima indicados, todas estão diretamente ligadas à produção de conhecimentos relativos à ciência da produção. 0 ponto máximo desse processo foi a constituição dos INSTITUTOS NACIONAIS DE CIÊNCIA E TECNOLOGIA, considerados como "programa top da ciência brasileira". Dentre os 144 INCTs aprovados com recursos públicos federais e estaduais, vários são relativos à nanotecnologia. 
Duas últimas ações do governo federal devem ser aqui indicadas. No ano de 2009 houve edital que implicou na construção de 16 redes de pesquisas de pequenas dimensões. No ano de 2011, pela primeira vez, foi aberto um edital para a constituição de redes de pesquisas em Nanotoxicologia. Como resultado temos constituídas 6 redes, com recursos aproximados de $\mathrm{R} \$ 1.500 .000,00$.

Portanto, depois de 10 anos de politicas públicas em nanotecnologia ainda não temos resultados obtidos neste campo da nanotoxicologia, pois as redes contempladas iniciaram seus trabalhos somente em 2011. Esse é outro fato que explicita a exclusão presente nessa política pública brasileira no que toca aos impactos da nanotecnologia, em especial no que toca aos impactos na saúde humana. Também aqui se pode fazer as mesmas contas para se determinar o quanto, percentualmente, se aplicou de recursos neste tema ao longo de 10 anos dessa política pública. Novamente, o resultado vai ser insignificante em termos percentuais.

A seguir vamos apresentar uma síntese dos objetivos dos diversos editais aqui apresentados e analisados. Isso nos dará elementos para analisar as concepções que consubstanciam essa política pública em nanotecnologia no Brasil. Os objetivos são os seguintes:

1) Incrementar o desenv científico e tecnológico;

2) Incrementar a competividade internacional da ciência, tecnologia e inovação brasileira;

3) Desenv regional igualitário;

4) Integração entre centro de pesquisas e empresas públicas e privadas;

5) Criação de empregos qualificados; 
6) Incrementar o nível tecnológico da indústria brasileira;

7) Incrementar o desenv. Econômico brasileiro

Esses objetivos estão devidamente alicerçados no discurso do presidente Lula proferido em Campinas/SP, no dia 18/08/05, por ocasião da instituição do Programa Brasileiro de Nanociência e Nanotecnologia. Os pontos centrais desse discurso são os seguintes:

1) Brasil necessita exportar conhecimentos;

2) Inovação tecnológica é a base para o novo brasil que queremos para o futuro;

3) Brasil é um país desigual. Ao mesmo tempo tem lugares na 1a․ Revolução industrial e na 3a․ Revolução industrial;

4) A comunidade científica deve ser a responsável pelas decisões de pesquisa;

5) Ciência e tecnologia são ferramentas essenciais para o desenvolvimento. Econômico e social e são prioridades do governo;

6) 0 melhor investimento é colocar dinheiro em ciência, tecnologia e educação;

7) Necessidade de incrementar a conecção entre universidade e empresas;

8) Programa de nanotecnologia é parte da política industrial, tecnológica e de comércio exterior

9) Poder = conhecimento científico e tecnológico

10) A melhor forma de olhar para a justiça social 


\section{N anotecnologia, agricultura e alimentos no Brasil}

No tópico anterior não há qualquer referência nas ações desenvolvidas pelo Ministério de Ciência, Tecnologia e Inovação - MCT (principal articulador do desenvolvimento das nanotecnologias no governo federal) que seja relativa a nanotecnologias aplicadas à agricultura e alimentos. Não há um edital específico para isso. Não há uma rede de pesquisas dedicada ao tema oriunda de algum edital para a constituição de redes de pesquisas em nanotecnologia. Não há qualquer Instituto Nacional de Ciência e Tecnologia - INCT (que são as instituições top da ciência brasileira) voltado a aplicações da nanotecnologia na agricultura, alimentos, indústria transformadora de alimentos.

Claro que, de maneira residual, poderá haver alguma aplicação nos campos acima indicados, decorrentes de conhecimentos produzidos pelos INCTs identificados. Isso é muito diferente de desenhar uma política de nanotecnologia voltada às questões postas pela segurança e soberania alimentar indicadas neste trabalho.

Portanto, o polo central do desenvolvimento das nanotecnologias no Brasil decidiu ao longo deste século deixar as questões de nanotecnologia, agricultura e alimentos por conta de outra instituição, no caso a EMBRAPA. Esta participou do edital Edital CNPq 29/2005, dedicado a constituição de 10 redes de pesquisas em Nanotecnologia. A EMBRAPA participou, mas não foi contemplada. A partir dessa constatação de que via MCT não iria sair recursos para o desenvolvimento de nanotecnologias voltadas à agricultura e alimentos, a EMBRAPA passou a se articular para que este desenvolvimento se desse com recursos diretamente captados pela própria instituição graças a sua articulação política interna ao governo federal. Recursos extra orçamentários foram fundamentais para a construção de prédios e aquisição de equipamentos na Embrapa Instrumentação de São Carlos/SP, local central da Embrapa que passou a 
ser o centro irradiador das ações desta instituição em termos de produção de conhecimentos nanotecnológicos aplicados ao Agronegócio.

É importante ressaltar que é esta denominação dada pela EMBRAPA ao seu principal laboratório na EMBRAPA INSTRUMENTAÇÃO é Laboratório Nacional de Nanotecnologia para o Agronegócio (LNNA). Não é laboratório Nacional de Nanotecnologia da Agricultura Familiar. Claro que isso significa a compreensão de que a nanotecnologia deve ser voltada ao agronegócio e não à agricultura familiar. Claro que essa redação não está escrita em documentos oficiais da EMBRAPA, mas a opção é clara.

Em folder produzido pela EMBRAPA (2010) e intitulado "Nanotecnologia, obter o máximo do mínimo", podemos resgatar algumas das concepções desta instituição sobre nanotecnologia e suas aplicações. No item do folder sobre nanotecnologia e agricultura somos informados que a EMBRAPA investe em Nanotecnologia desde 1996.

A seguir somos informados que o Laboratório Nacional de Nanotecnologia para o Agronegócio - LNNA - criado em 2009, foi financiado pela FINEP, órgão do MCT, o que indica que isto não se deu via edital e sim via ações políticas da EMBRAPA para a conquista de recursos extra orçamentário.

As linhas de pesquisas da EMBRAPA relativas à Nanotecnologia estão assim descritas no folder: 
As principais linhas de pesquisas contempladas pelo LNNA incluem:

- Desenvolvimento de sensores e biossensores, aplicados ao controle de qualidade, cercação e rastreabilidade de bebidas e alimentos;

- Fabricaçăo de novos materiais de fonte renovável, como polímeros naturais e nanobiocompósitos com propriedades específicas;

- Membranas de biopolímeros e revestimentos poliméricos comestíveis para preservação de alimentos;

- Filmes finos e superfícies para fabricação de embalagens inteligentes, comestíveis e superficies ativas;

- Nanopartículas, compósitos e fibras para o desenvolvimento de materiais reforçados biodegradáveis usando produtos naturais como fibras de sisal, juta, coco, bagaço de cana, curauá e outras para aplicações industriais;

- Nanopartículas orgânicas e inorgânicas para liberação controlada de nutrientes e pestici das em solos e plantas, de fármacos para uso veterinário;

- Caracterização de materiais de interesse do agronegócio para obtençāo de informaçōes inéditas sobre partículas de solos e plantas, bactérias e patógenos de interesse agrícola;

- Estudos nanotoxicológicos e de impactos na saúde e meio ambiente para garantir o desenvolvimento de nanomateriais de forma segura e responsável.

Fonte: imagem extraída de parte do folder "Nanotecnologia, obter o máximo do mínimo", Embrapa (2010)

Quem trabalha nessas linhas de pesquisas são os componentes da Rede de Pesquisas em nanotecnologia para o agronegócio, criada em 2006, com 150 pesquisadores de 53 instituições (14 unidades da EMBRAPA e 39 centros acadêmicos de excelência).

Atualmente, a EMBRAPA vem desenvolvendo pesquisa com sensores nanotecnológicos, em que o exemplo mais divulgado e conhecido é a chamada "língua eletrônica" aplicada a determinar a qualidade do café, mas que pode ser empregada a determinar a qualidade de outros líquidos.

Também atua na produção de embalagens ativas e coberturas comestíveis para a preservação de frutas e hortaliças. Aqui o caso divulgado como de sucesso dessas pesquisas se trata do nanofilme que envolve a maçã e com isto consegue sua conservação por um tempo maior, combatendo assim o desperdício desta fruta.

Como desafios a serem conquistados, esse folder nos indica a produção de nanofibras para serem inseridas em polímeros para 
aplicações industriais, nanocatalizadores para a degradação de poluentes em águas, sistemas de liberação controladas de insumos via hidro géis e nanoparticulas poliméricas que poderão ser usadas na aplicação de fertilizantes e agroquímicos.

\section{Conclusões preliminares para o debate público}

O desenvolvimento das nanotecnologias no Brasil vem sendo capitaneado pelo Ministério de Ciência, Tecnologia e Inovação MCTI. Os demais órgãos do governo federal vêm a reboque das ações deste ministério. No MCTI a visão dominante é a de que "as novas tecnologias levam a inovações; estas necessariamente implicam aumento da competitividade de empresas, indústrias, países, o que, por sua vez, assegura o crescimento econômico, que vai redundar em mais bem-estar social". Portanto, a visão hegemônica atribui uma causação linear entre as variáveis, configurando o chamado modelo linear de inovação.

Já em 2007, Martins et al. (cood) afirmavam que o desenvolvimento das nanociências e Nanotecnologias no Brasil, portanto, nasceu e permanece até o presente sob a égide de que não deve haver controle social sobre ele. Quem deve decidir são

os especialistas no assunto, o Estado - mais especificamento o MCT - e segmentos empresariais que conseguem acesso a conselhos e/ou decisões ministeriais, Esta é a concepção do desenvolvimento da nanociência e nanotecnologia no Brasil que se inicia com o Edital CNPq Nano m.01/2001 e se perpetua nos demais editais voltados a área (Martins, 2007, p. 14).

Embora seja público que as pesquisas no Brasil são realizadas com recursos públicos - em especial as relativas à nanociência e nanotecnologia - oriundos dos impostos pagos pela sociedade, os atores e agentes que contribuem e decidem os rumos do 
desenvolvimento das nanociências e nanotecnologias no Brasil não abarcam os atores e agentes sociais tais como entidades representativas da agricultura familiar, de defesa do interesse difuso da sociedade (meio ambiente, saúde, consumidor) entidade representativa dos trabalhadores (centrais sindicais, sindicatos e seus órgão de assessoria), entidade de defesa dos direitos humanos, entidades de defesa da participação popular, entidades religiosas, etc.

Portanto, esse é o cenário político em que se encontram as ações desenvolvidas pelo MCT. Nelas, não estão no horizonte desenvolver uma politica de desenvolvimento das nanotecnologias voltadas à segurança e soberania alimentar. Isso é uma não questão para o MCTI.

Sobram então as ações da EMBRAPA. Claro que ela vem se consolidando como "proprietária do tema" nanotecnologia aplicada à agricultura e alimentos. Trata-se, portanto, de examinar o que a EMBRAPA vem fazendo em relação às nanotecnologias. Como foi demonstrado ela optou claramente pelo agronegócio, tanto assim que seu principal laboratório de nanotecnologia leva o nome de Laboratório Nacional de Nanotecnologia para o Agronegócio.

Embora aqui se reconheça a necessidade de se realizar uma pesquisa que apure de forma mais exata qual tem sido a produção de conhecimentos sobre nanotecnologia produzida pela EMBRAPA, via os papers apresentados nos seminários da rede EMBRAPA de nanotecnologia - são estes conhecimentos produzidos dirigidos ao agronegócio ou à agricultura familiar - poderão apresentar de maneira mais clara o caminho adotado pela EMBRAPA no desenvolvimento das nanotecnologias aplicadas à agricultura e alimentos.

O debate sobre se as prioridades das ações da EMBRAPA de maneira geral contemplam mais o agronegócio ou a agricultura 
familiar é presente nos estudos relativos a contribuição da EMBRAPA no desenvolvimento agrícola brasileiro. Não é objetivo deste texto reproduzir aqui esse debate e avaliação em termos gerais. 0 objetivo aqui é indicar que no caso das nanotecnologias aplicadas à agricultura e alimentos a hipótese de trabalho é de que a visão hegemônica na EMBRAPA sobre nanotecnologia é que as nanotecnologias devem estar diretamente ligadas a processos que estão em curso há décadas no sentido de intensificar o processo de "industrialização da agricultura". A nanotecnologia vem como a plataforma para o estabelecimento de um novo paradigma do sistema agroalimentar dominantes nesta globalização dos mercados de produtos/commodities agrícolas. A EMPRAPA procura estar sintonizada com esse processo atuando nessa área de fronteira de conhecimento. Isso significa priorizar suas ações em nanotecnologia voltadas para esse processo hegemonizado pelo agronegócio e não pela agricultura familiar.

É preciso salientar que o processo final de "industrialização da agricultura" poderá ser alcançado via as nanotecnologias denominadas de "nanofabricação". Se tudo o que conhecemos e utilizamos são materializados em termos de átomos e moléculas concatenados, se dominamos as tecnologias de concatenar os átomos e moléculas que significam, materializam o café, não será mais necessário o cultivo do café nos moldes que conhecemos hoje. Isso terá implicações imensas do ponto de vista social, econômico, de comercio internacional, etc. 0 mesmo se aplica às outras culturas hoje conhecidas.

Portanto, o desenvolvimento das nanotecnologias - no Brasil e no exterior - está diretamente ligado ao futuro de milhões, bilhões de agricultores que poderão até mesmo serem eliminados dos mercados com o advindo da "nanofabricação". Este artigo tem por objetivo iniciar o debate no Brasil sobre as consequências do desenvolvimento das nanotecnologias na agricultura brasileira, na agricultura familiar, nas políticas públicas relativas à segurança e soberania alimentar. Esperamos que este "ponta 
pé inicial" possa despertar a atenção de outros pesquisadores e com isto tornar esse uma tema de pesquisas para os pesquisadores de desenvolvimento agrícola no Brasil.

\section{Ref erências}

BRASIL. Ministério da Ciência e Tecnologia. Conselho Nacional de Desenvolvimento Científico e Tecnológico. Edital CNPq Nano ${ }^{\circ}$ 01/2001. Brasília, 2001. Disponível em: <http://www.memoria.cnpq.br/servicos/editais/ct/nanociencia.htm>. Acesso em: 11 jan. 2007.2

Carlos Mieletz (org). Desenvolvimento Agricola e Questão Agrária. São Paulo: editora Fundação Perseu Abramo, 2013

EMBRAPA INSTRUMENTAÇÃO. Nanotecnologia, obter o máximo do mínimo. Embrapa Instrumentação: São Carlos, Julho 2010.

ETCGROUP. Nanotecnologia: Os riscos da tecnologia do futuro. Porto Alegre: L\&PM Editores, 2005.

HOSSAM, Haick. Nanotecnology and Nanosensors. Israel Institute of Technology. 2013.

International Center For Technology Assessment. Principles of the Oversight in Nanotechnologies and Nanomaterials. ICTA, Washongton, 2007.

MALUF, Renato S. A agricultura e a promoção da soberania e segurança alimentar e nutricional: entraves e desafios. In: MIELETZ, Carlos (org). Desenvolvimento Agrícola e Questão Agrária. São Paulo: Fundação Perseu Abramo, 2013

MARTINS, Paulo R. O Necessário Confronto Social. In: Scientific American Brasil. São Paulo, Ano 3 N32, Jan. 2005, p.26

. (coord) Nanotecnologia, Sociedade e Meio Ambiente. 1 Seminário Internacional. São Paulo, Associação Editora Humanitas, 2005, 285p . Introdución a la nanotecnología: un análisis sociológico. Arxius de Ciencies Socials. Facultat de Ciencies Socials. Valencia, Espanha, N.12/12 Desembre/2005

(Org). Nanotecnologia, Sociedade e Meio Ambiente. Trabalhos apresentados no segundo seminário Internacional. São Paulo: Xamã Editora, 2006. 
. Nanotecnologia e meio ambiente para uma sociedade sustentável. In: MARTINS, Paulo R. (org). Nanotecnologia, Sociedade e Meio Ambiente. Trabalhos apresentados no segundo seminário Internacional. São Paulo: Xamã Editora, 2006.

. Nanotecnologia. In CATTANNI, Antonio D e HOLZMANN, Lorena. Dicionário de Trabalho e Tecnologia. Porto Alegre: UFRGS Editora, 2006. lo: IIEP, 2006.

. Nanotecnologia. In: Nanotecnologia e os trabalhadores. São PauMARTINS, Paulo R. (coord) et al. Revolução Invisível. Desenvolvimento recente da nanotecnologia no Brasil. São Paulo: Xamã Editora, 2007.

. Nanotecnologia, Sociedade e Meio Ambiente em São Paulo, Minas Gerais e Distrito Federal. São Paulo: Xamã Editora, 2007.

MARTINS, Paulo R; BRAGA, Ruy. Promessas e dilemas da revolução invisível. In: Sociologia, Ano I número 5. São Paulo: Editora Scala, 2007.

. A tecnociência financeirizada: dilemas e riscos da nanotecnologia. In: Universidade e Sociedade. São Paulo, Ano XVII, N.40, Julho de 2007, p139-147 MARTINS, Paulo R. Nanotecnologia, uma introdução. In: EMERICK, Maria C et al. (orgs). Novas Tecnologias na Genética Humana: Avanços e Impactos para a Saúde. Rio de Janeiro: Gestec, 2007.

Desenvolvimento Recente da Nanotecnologia no Brasil: Reflexões sobre a política de riscos e impactos ambientais, sociais e econômicos em $\mathrm{Na}$ notecnologia In: EMERICK, Maria C et all (orgs). Novas Tecnologias na Genética Humana: Avanços e Impactos para a Saúde. Rio de Janeiro: Gestec, 2007.

. Contribuição da Renanosoma ao Debate sobre Ética e Nanotecnologia no Brasil. In: SARTI, Flávia Mori; SANTOS, Gislene Aparecida dos (org). Ética, Tecnologia e Comunicação. Rio de Janeiro: Rubio, 2009.

MARTINS, Paulo; DULLEY, Richard D. (Orgs). Nanotecnologia, Sociedade e Meio Ambiente. Trabalhos apresentados no terceiro seminário internacional. São Paulo: Xamã Editora, 2008.

MARTINS, Paulo R. Et all. Actividades relacionadas com las nanotecnologias em Brasil. In: FOLADORI, Guillermo; INVERNIZZI, Noela. Nanotecnologias em América Latina. Mexico: Univ Autonoma de Zacatecas, 2008.

. Developing Strategies in Brazil to Manage the Emerging Nanotechnology and its Associated Risks. In: LINKOV, Igor; STEEVENS, Jeffrey. Nanomaterials: Risk and Benefits. Dordrecht, 2008 
MARTINS, Paulo R; RAMOS, Soraia F et all. Impactos das nanotecnologias na cadeia de produção da soja brasileira. São Paulo: Xamã Editora, 2009.

MORIN, edgard. Por uma globlalização plural. Folha de São Paulo domingo, 31/3/02, p.a16.

NANOPART Res, ROCO, Mihail C et al. Global nanotechnology development from 1991 to 2012: patents, scientific publications, and effect of NSF funding, 2013.

NATIONAL NANOTECHNOLOGY INITIATIVE. National Nanotechnology Initiative Supplement to the President's 2014 Budget,NNI. Washington, 2013

PREMEBIDA, Adriano. Biotecnologias: Dimensões sociológicas e Políticas. São Paulo: Parco Editorial, 2011.

Programa Nanotecnologia do avesso N. 199. Entrevistado Evgeny Klochikheim. Disponível em: www.nanotectnologiadoavesso.org

Recebido em 05/05/2016

Aprovado em 27/06/2016 